\title{
Association of Temporomandibular Disorders Symptoms with Anxiety and Depression in Tunisian Patients
}

Aymen Ben Hadj Khalifa ${ }^{1,2,3 *}$, Ghada Ayari ${ }^{1,4}$, Monia Elhadj Khalifa ${ }^{5}$,Walid Ghorbel ${ }^{6,7}$ Chiraz Baccouche ${ }^{1,2,9}$, Soumaya Touzi ${ }^{2}$ Faten Ben Amor $^{7,8}$, Hichem Ghedira ${ }^{1,3}$

${ }^{1}$ Biological and Clinical Dento-Facial Approach Laboratory (ABCDF) LR12ES10, Faculty of Dental Medicine, University of Monastir, Monastir, Tunisia

${ }^{2}$ Department of Dental Anatomy, Faculty of Dental Medicine, University of Monastir, Monastir, Tunisia

${ }^{3}$ Department of Pediatric Dentistry, Faculty of Dental Medicine, University of Monastir, Monastir, Tunisia

${ }^{4}$ Department of Restorative Dentistry-Endodontics, Faculty of Dental Medicine, University of Monastir, Tunisia

${ }^{5}$ Psychiatrist practicing in Paris hospitals, Ancient Assistant, Department of Psychiatry, University Hospital of Monastir, Tunisia

${ }^{6}$ Department of Stomatology, Hedi Chaker University Hospital, Sfax, Tunisia

${ }^{7}$ Department of Anatomy, Faculty of Dental Medicine, University of Monastir, Monastir, Tunisia

${ }^{8}$ Outpatient Department, University Dental Clinic, Faculty of Dental Medicine, University of Monastir, Monastir, Tunisia

${ }^{9}$ Department of Stomatology, Taher Sfar University Hospital, Mahdia, Tunisia

\section{Article History \\ Received: 04.02.2021 \\ Accepted: 17.02 .2021 \\ Published: 03.03.2021}

Journal homepage:

https://www.easpublisher.com

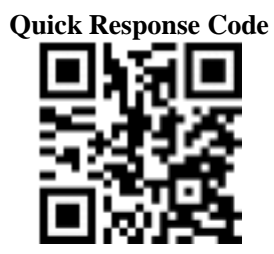

Abstract: Aim: The aim of this study was to assess the prevalence of temporomandibular disorders (TMD) and their association with anxiety and depression in Tunisian patients. Methods: Two hundred patients were enrolled in the study and completed a socio-demographic survey. Then, TMD and psychological distress were assessed. Descriptive statistics and logistic regression analysis were performed with TMD symptoms as the outcome variable. Results: The prevalence of patients with at least one TMD symptom was $29 \%$. Almost two-thirds of subjects (64\%) experienced a history of psychological stress. Anxiety symptoms were determined in $58 \%$ of patients, while depression symptoms were found in $23.5 \%$ of patients. Logistic regression analysis showed that gender, anxiety, depression and stress were significantly related to TMD symptoms. Conclusion: The prevalence of TMD symptoms in Tunisian patients is not very high. Psychological distress; anxiety, depression and stress are correlated with TMD. Risk factors for patients with confirmed TMD were anxiety, depression, stress and female sex. Thus, further longitudinal research of the causality is warranted.

Keywords: Temporomandibular Disorders, Anxiety, Depression, Prevalence, Correlation, Study.

Copyright $\odot$ 2021 The Author(s): This is an open-access article distributed under the terms of the Creative Commons Attribution 4.0 International License (CC BY-NC 4.0) which permits unrestricted use, distribution, and reproduction in any medium for non-commercial use provided the original author and source are credited.

\section{INTRODUCTION}

Temporomandibular disorders (TMD) is an all-inclusive term referring to a heterogeneous group of neuro-musculoskeletal and/or psycho-physiologic disorders involving temporomandibular joints (TMJ), masticatory muscles, and associated tissues [1-3]. The common characteristics are orofacial pain, essentially, in the temporomandibular joint (TMJ) region, masticatory dysfunction, limitation of mandible movement, and the presence of a clicking sound in the TMJ [3-5].

The etiology of TMD is considered to be multifactorial [3-7]. It is directly related to harmful habits, psychological factors, neuromuscular factors, trauma, dysfunctions of the masticatory musculature and adjacent structures, occlusal interferences, malpositioning or loss of teeth, postural changes, extrinsic and intrinsic changes of TMJ structure, nonfunctional movements of the mandible (bruxing), tooth clenching habits, or a combination of such factors. However, the relative importance of individual factors is still controversial.

Stress, fatigue, anxiety, depression, sleep disorders, and a fast paced life affect negatively the human psyche [4]. Psychological factors are known to play a role in the etiology and persistence of TMD [5, 8]. As with most pain disorders, a high prevalence of depression and anxiety is associated with TMD [9,10]. 
This study aimed at determining the prevalence of TMD in Tunisian subjects. It also investigated its-correlation with psychological factors such as anxiety and depression.

\section{Material AND Methods}

It was a cross-sectional study carried out in the consultation of the University Dental Clinic of Monastir (Tunisia). The sample was considered of 200 subjects randomly selected. Participation in the study was offered during the consultations in an informed way. The patients were free to accept or to refuse. Subjects voluntarily agreed to answer the form, which did not influence the patient's medical management.

\section{Inclusion criteria}

To be included in the study, patients had to be 16 years or older and be able to complete the forms correctly.

\section{Non-inclusion criteria}

Patients who consult for emergencies. Uneducated patients or unable to self-complete the questionnaire.

\section{Data Collection}

Data were collected using a questionnaire and an oral examination. The questionnaire included the socio-demographic data of the patients and the scale of the evaluation of anxiety and depression.

The level of anxiety and depression was selfrated by the Hospital Anxiety and Depression Scale (HADS) originated from the study of Zigmond and Snaith [11]. It is composed of a 14-item selfadministered rating scale consisting of two subscales: seven items measuring anxiety disorders and seven items measuring depression. The individual items are each rated on a four-point scale (0-3) resulting in maximum subscale scores of 21 , with higher scores indicating greater levels of distress. It was decided that a score strictly greater than seven $(>7)$ present on both subscales indicates a symptomatology of anxious and depressive state. The French and Arabic version of the HADS was used for assessment of anxiety and depression levels.

Examination for TMD was performed according to the Research Diagnostic Criteria for Temporomandibular Disorders (RDC/TMD) developed by Dworkin and LeResche in 1992 [10]. An oral examination of teeth and TMJ was made, based on selfreport and clinical criteria, each patient who mentions at least a notion of articulo-muscular pain and/or discomfort is retained as a patient with TMD.

\section{Statistical Analysis}

Statistical analyses were performed using SPSS 23.0. T-student test and Chi-square test were used to assess the association between TMD and the different characteristics of the sample. Significance was considered for $\mathrm{P}<0.05$.

\section{ReSUlts}

The mean age of the included participants was 31.4 years $(S . D=13)$ with extremes ranging from 16 to 77 years. The most represented age group was 21-30 years old with $47.5 \%$ (Table1).

A female predominance was observed $59.5 \%$ against $40.5 \%$ men, with a sex ratio equal to 0.68 . (Table1).

In all, $46.5 \%$ were students, $37.5 \%$ were in professional activity, $13.5 \%$ were unemployed and $2.5 \%$ were retired (Table 1 ).

Among participants, $64 \%$ reported a history of psychological stress in inducing and increasing complaints (Table 3).

The prevalence of anxiety symptoms was determined in $58 \%$ of patients, while of the depression symptoms was found in $23.5 \%$ of patients. The prevalence of TMD was $29 \%$ (58 patient).

An association was found between TMD and sex (Table 2). A statistically significant relationship between the presence of TMD symptoms and all psychological factors was found (Table 3).

Table-1: Distribution of patients regarding age, gender and occupation

\begin{tabular}{|c|c|c|}
\hline Variables & Number & $\%$ \\
\hline \multicolumn{3}{|c|}{ Age group (years) } \\
\hline$\leq 20$ & 29 & 14.5 \\
\hline 21 to 30 & 95 & 47.5 \\
\hline 31 to 40 & 37 & 18.5 \\
\hline 41 to 50 & 20 & 10 \\
\hline 51 to 60 & 9 & 4.5 \\
\hline$>60$ & 10 & 5 \\
\hline \multicolumn{3}{|c|}{ Gender } \\
\hline Female & 119 & 59.5 \\
\hline Male & 81 & 40.5 \\
\hline Occupation & 93 & 46.5 \\
\hline Students & 75 & 37.5 \\
\hline In activity & 27 & 13.5 \\
\hline Unemployed & 5 & 2.5 \\
\hline Retirement & 200 & 100 \\
\hline Total &
\end{tabular}


Table-2: Association between Demographic characteristics (age, gender) and TMD

\begin{tabular}{|c|c|c|c|c|}
\hline \multirow{2}{*}{ Sex * } & Females (119) & With TMD (58) & Without TMD (142) & P \\
\cline { 2 - 4 } & Males (81) & 38 & 81 & \multirow{2}{*}{$0.041 *$} \\
\hline \multirow{2}{*}{ Age } & $\leq 32(135)$ & 20 & 61 & \multirow{2}{*}{0.913} \\
\cline { 2 - 5 } & $>32(65)$ & 40 & 95 & 47 \\
\hline \multicolumn{2}{|c|}{$*$ Relative Risk (RR) = 1.29: Females have a higher risk of developing TMD compared to males. } \\
\hline
\end{tabular}

Table-3: Association between TMD and anxiety, depression and stress

\begin{tabular}{|c|c|c|c|c|}
\hline \multicolumn{2}{|c|}{} & With TMD (58) & Without TMD (142) & \multirow{2}{*}{ P } \\
\hline \multirow{2}{*}{ Anxiety State } & Yes (116) & 49 & 67 & \\
\cline { 2 - 4 } & No (84) & 9 & 75 & \multirow{2}{*}{$\mathbf{0 . 0 2 2}^{*}$} \\
\hline \multirow{2}{*}{ Depression State } & Yes (47) & 22 & 25 & \\
\cline { 2 - 4 } & No (153) & 36 & 117 & \multirow{2}{*}{$\mathbf{0 . 0 2 6}^{*}$} \\
\hline \multirow{2}{*}{ Stress } & Yes (128) & 44 & 58 & \\
\cline { 2 - 4 } & No (72) & 14 & & \\
\hline
\end{tabular}

\section{DiscuSSION}

The prevalence of anxiety and depression in our study were respectively $58 \%$ and $23.5 \%$. Bonjardim et al. [4] used the same scale and reported anxiety and depression in $16.58 \%$ and $26.71 \%$ of Brazilian subjects, respectively. In Chinese adolescents [6], the prevalence of anxiety and depression was higher than those of this study, respectively $65.2 \%$ and $34.9 \%$. These results show that there is evidence of psychological disturbances in the general population and specifically in those who consult in dentistry, as verified also by Tiller [12] and Zinke \& col. [13]

In this study, $29 \%$ of Tunisian subjects presented one or more TMD symptoms. A few of these patients had prior knowledge of TMD. These results were in accordance with those found in the study of Rokaya et al. [5] which reported a prevalence of $30.6 \%$ among Nepalese subjects. Likewise, Fernandes Azevedo [7] found that TMD was present in 36.2\% of subjects who were students. In another study [6], the prevalence of Asian adolescents with at least one TMD symptom was $61.4 \%$. However, other studies reported an even higher prevalence of TMD values up to $77 \%$ [2, 8, 14-19], especially among those evaluating students.

These differences in the reported prevalence may be due to the characteristics of the study process, the characteristics of the populations, the biological differences, the psychosocial factors and the time when the questionnaire was administered.

The prevalence of TMD in the general population, based on RDC/TMD [10] showed that females had four times greater risk than males. Earlier studies of various populations [2, 14, 18, 19] found that women had a higher prevalence of TMD than men, as well as the findings in our study $(\mathrm{p}=0.041$, Relative Risk=1.29). Along the same lines, other studies showed that the most important risk factor for developing TMD is female gender [20-22]. In addition, Auerbach et al.
[23] found that female TMD patients were significantly more depressed than males.

The high prevalence of TMD in women might be related to physiological characteristics, particularly hormonal variations and structures in connective tissue and muscle. The greater laxity of these tissues, which is related to estrogen level, may explain why these tissues are less able to support functional pressure, thereby leading to TMD [16]. LeResche et al. [24] found that pain intensity varied during the menstrual cycle among women with TMD: pain intensity was greater when estrogen concentration was higher.

This association between TMD and gender was not significant in others studies like the one of Lei [6] among Chinese adolescents and Bonjardim in a group of students [19].

In this study, $64 \%$ of subjects presented a psychological stress which is similar to the results reported by other studies [5, 15, 25]. These studies showed an association between TMD and mental anxiety or stress. Also, the findings of Auerbach [23] are consistent with previous research indicating a link between emotional dysfunction and TMD. They are supportive of the conclusion that psychological factors play a more pronounced role when there is pain. List et al. [26] verified a higher degree of stress and behavior problems in adolescents with TMD than in healthy individuals.

According to this study, a significant association was found between TMD and anxiety $(\mathrm{p}<0.001)$, depression $(\mathrm{p}=0.022)$ and stress levels $(\mathrm{p}=$ 0.026). These results are accordance with those corroborate $[2,4-6,9]$.

In adolescents with TMD, psychosocial factors such as increased stress levels, anxiety, depression, somatic complaints, and emotional problems seem to play a more prominent role than dental factors. 
Individuals subject to stress may develop parafunctional habits and muscle tension, which lead to the development of TMD [16]. Thus, parafunctional components, especially those that increase muscle tension, and changes in emotional states are good indicators of jaw pain in people with TMD, which suggests that anxiety and depression are etiological factors in TMD. In his study on Portuguese patients, Minghelli [16] found a high prevalence of TMD, which was significantly associated with anxiety and depression. Vassend et al. [27] found a strong correlation between trait anxiety scores and TMD pain. Liao et al. [18] found that TMD incidence in individuals with depression was 2.65 times that of those without depression. Counter to the study of Bonjardim [19], TMD was found to have a statistically significant association with anxiety but not with depression.

Anxiety and depression may be an important factor in the perception of pain. This evidence was confirmed by other studies [28-30] which indicated that anxiety and depression are related to increased pain reports in clinical settings. Other researches [22, 23, 31] suggested a strong association between muscular pain and anxiety symptoms, while depressive symptoms may be specific to the development of joint pain.

Otherwise, some studies like the one of Spruijt [32] did not found an association between anxiety, depression and TMD. Also, Azevedo [7] found that joint TMD was not associated with anxiety levels.

Greater academic pressures lead to higher levels of psychological distress generating anxiety and stress, which in turn results in increased TMD symptoms [19]. Ahmed K. Ibrahim [33], through a systematic review, found that university students are most at risk of depression. In this study, despite a large number of young students mainly between 21-30 years, there was no relationship between age and psychiatric diseases. Diraçoğlu [21] also proves the absence of this relationship.

Studies have shown that TMD patients have higher levels of psychological and affective distress, and validated the important role that psychosocial factors play in predisposing, precipitating, and perpetuating TMD $[6,31]$.

An advantage of this study is the use of an instrument with very good statistical properties for anxiety and depression HADS [11]. According to its developers, HADS was conceived for use in nonpsychiatric hospital departments or in the general population to screen patients for the two most common well-known mental illnesses. It has been shown that its sensitivity, specificity and validity were good [4, 20, 21, 34]. It can be used effectively as an initial screening instrument for the most common manifestations of anxiety and depression [35]. Its purpose is not to discriminate the various types of depression or anxiety states, nor their secondary or primary nature, but to identify the existence of symptomatology and assess its severity.

In our diagnosis, the possibility of a TMD misdiagnosis cannot be excluded, especially for jointrelated pathology. Thus, a more detailed clinical examination of TMD signs and symptoms and imaging tests to confirm the diagnosis would have been useful.

This study did not take into consideration general diseases, alcohol addiction, dental treatments, occlusal factors, bruxism and their relationship with TMD and psychological distress [5, 36, 37]. Therefore, this study may be extended to see the correlations of TMD with the previously mentioned factors. Nevertheless, this work cannot be declared free from patient selection bias. It does not represent the general population but provides a representative figure.

\section{CONCLUSION}

The findings of this study are consistent with previous research indicating a link between psychological factors and TMD. Risk factors for patients with confirmed TMD were anxiety, depression, stress and female sex.

This was a pioneer study; thus, actual TMD prevalence might be higher. A greater understanding of this condition among populations exposed to high levels of emotional stress, such as college students, is of considerable importance in increasing awareness of TMD signs and symptoms and encouraging affected individuals to seek treatment. It is important that TMD be diagnosed early to prevent future complications.

\section{ACKNOWLEDGEMENTS}

Special thanks should be given to Professor Yamina Elelmi from the Faculty of Dental Medicine of Monastir, Tunisia, for her help and assistance in this work.

\section{REFERENCES}

1. Tay, K. J., Yap, A. U. J., Wong, J. C. M., Tan, K. B. C., \& Allen, P. F. (2019). Associations between symptoms of temporomandibular disorders, quality of life and psychological states in Asian Military Personnel. Journal of oral rehabilitation, 46(4), 330-339.

2. Wieckiewicz, M., Grychowska, N., Wojciechowski, K., Pelc, A., Augustyniak, M., Sleboda, A., \& Zietek, M. (2014). Prevalence and correlation between TMD based on RDC/TMD diagnoses, oral parafunctions and psychoemotional stress in Polish university students. BioMed research international, 2014. 
3. American Society of Temporomandibular Joint Surgeons. (2003). Guidelines for diagnosis and management of disorders involving the temporomandibular joint and related musculoskeletal structures. Cranio, 21, 68-76.

4. Bonjardim, L. R., Gavião, M. B. D., Pereira, L. J., \& Castelo, P. M. (2005). Anxiety and depression in adolescents and their relationship with signs and symptoms of temporomandibular disorders. International Journal of Prosthodontics, 18(4).

5. Rokaya, D., Suttagul, K., Joshi, S., Bhattarai, B. P., Shah, P. K., \& Dixit, S. (2018). An epidemiological study on the prevalence of temporomandibular disorder and associated history and problems in Nepalese subjects. Journal of dental anesthesia and pain medicine, 18(1), 27.

6. Lei, J., Fu, J., Yap, A. U., \& Fu, K. Y. (2016). Temporomandibular disorders symptoms in Asian adolescents and their association with sleep quality and psychological distress. CRANIO®, 34(4), 242249.

7. Fernandes Azevedo, A. B., Câmara-Souza, M. B., Dantas, I. D. S., de Resende, C. M. B. M., \& Barbosa, G. A. S. (2018). Relationship between anxiety and temporomandibular disorders in dental students. CRANIO®, 36(5), 300-303.

8. Manfredini, D., Bandettini di Poggio, A., Cantini, E., Dell'Osso, L., \& Bosco, M. (2004). Mood and anxiety psychopathology and temporomandibular disorder: a spectrum approach. Journal of oral rehabilitation, 31(10), 933-940.

9. Rajan, R., \& Sun, Y. M. (2017). Reevaluating antidepressant selection in patients with bruxism and temporomandibular joint disorder. Journal of Psychiatric Practice®, 23(3), 173-179.

10. LeResche, L., \& Von Korff, M. R. (1992). Research diagnostic criteria for temporomandibular disorders: review, criteria, examinations and specifications, critique. $J$ Craniomandib Disord, 6(4), 301-355.

11. Zigmond, A. S., \& Snaith, R. P. (1983). The hospital anxiety and depression scale. Acta psychiatrica scandinavica, 67(6), 361-370.

12. Tiller, J. W. (2013). Depression and anxiety. The Medical Journal of Australia, 199(6), S28-S31.

13. Zinke, A., Hannig, C., \& Berth, H. (2019). Psychological distress and anxiety compared amongst dental patients-results of a cross-sectional study in 1549 adults. BMC oral health, 19(1), 1-6.

14. Oliveira, A. S. D., Dias, E. M., Contato, R. G., \& Berzin, F. (2006). Prevalence study of signs and symptoms of temporomandibular disorder in Brazilian college students. Brazilian oral research, 20(1), 3-7.

15. de Melo Rocha CO, Peixoto RF, de Resende CM, de Medeiros Alves AC, da Costa Oliveira ÂG, Seabra Barbosa GA. Psychosocial aspects and temporomandibular disorders in dental students. Quintessence International. 2017 Mar 1;48(3).
16. Minghelli, B., Morgado, M., \& Caro, T. (2014). Association of temporomandibular disorder symptoms with anxiety and depression in Portuguese college students. Journal of oral science, 56(2), 127-133.

17. Progiante, P. S., Patussi, M., Lawrence, H., Goya, S., Grossi, P. K., \& Grossi, M. L. (2015). Prevalence of Temporomandibular disorders in an adult brazilian community population using the research diagnosis criteria (axis I and II) for temporomandibular disorders (teh Maringa Study). The International journal of prosthodontics.

18. Liao, C. H., Chang, C. S., Chang, S. N., Lane, H. Y., Lyu, S. Y., Morisky, D. E., \& Sung, F. C. (2011). The risk of temporomandibular disorder in patients with depression: a population-based cohort study. Community dentistry and oral epidemiology, 39(6), 525-531.

19. Bonjardim, L. R., Lopes-Filho, R. J., Amado, G., Albuquerque, R. L., \& Goncalves, S. R. (2009). Association between symptoms of temporomandibular disorders and gender, morphological occlusion, and psychological factors in a group of university students. Indian Journal of dental research, 20(2), 190.

20. Giannakopoulos, N. N., Keller, L., Rammelsberg, P., Kronmüller, K. T., \& Schmitter, M. (2010). Anxiety and depression in patients with chronic temporomandibular pain and in controls. Journal of dentistry, 38(5), 369-376.

21. Dıraçoğlu, D., Yıldırım, N. K., Saral, I., Özkan, M., Karan, A., Özkan, S., \& Aksoy, C. (2016). Temporomandibular dysfunction and risk factors for anxiety and depression. Journal of back and musculoskeletal rehabilitation, 29(3), 487-491.

22. Velly, A. M., Gornitsky, M., \& Philippe, P. (2003). Contributing factors to chronic myofascial pain: a case-control study. Pain, 104(3), 491-499.

23. Auerbach, S. M., Laskin, D. M., Frantsve, L. M. E., \& Orr, T. (2001). Depression, pain, exposure to stressful life events, and long-term outcomes in temporomandibular disorder patients. Journal of oral and maxillofacial surgery, 59(6), 628-633.

24. LeResche, L., Mancl, L., Sherman, J. J., Gandara, B., \& Dworkin, S. F. (2003). Changes in temporomandibular pain and other symptoms across the menstrual cycle. Pain, 106(3), 253-261.

25. Habib, S. R., Al Rifaiy, M. Q., Awan, K. H., Alsaif, A., Alshalan, A., \& Altokais, Y. (2015). Prevalence and severity of temporomandibular disorders among university students in Riyadh. The Saudi dental journal, 27(3), 125-130.

26. List, T., Wahlund, K., \& Larsson, B. (2001). Psychosocial functioning and dental factors in adolescents with temporomandibular disorders: a case-control study. Journal of orofacial pain, 15(3).

27. Vassend, O., Krogstad, B. S., \& Dahl, B. L. (1995). Negative affectivity, somatic complaints, and 
symptoms of temporomandibular disorders. Journal of psychosomatic research, 39(7), 889-899.

28. de Leeuw, R., Studts, J. L., \& Carlson, C. R. (2005). Fatigue and fatigue-related symptoms in an orofacial pain population. Oral Surgery, Oral Medicine, Oral Pathology, Oral Radiology, and Endodontology, 99(2), 168-174.

29. Ferrando, M., Andreu, Y., Galdón, M. J., Durá, E., Poveda, R., \& Bagán, J. V. (2004). Psychological variables and temporomandibular disorders: distress, coping, and personality. Oral Surgery, Oral Medicine, Oral Pathology, Oral Radiology, and Endodontology, 98(2), 153-160.

30. Mongini, F., Ciccone, G., Ceccarelli, M., Baldi, I., \& Ferrero, L. (2007). Muscle tenderness in different types of facial pain and its relation to anxiety and depression: a cross-sectional study on 649 patients. Pain, 131(1-2), 106-111.

31. Kindler, S., Samietz, S., Houshmand, M., Grabe, H. J., Bernhardt, O., Biffar, R., ... \& Schwahn, C. (2012). Depressive and anxiety symptoms as risk factors for temporomandibular joint pain: a prospective cohort study in the general population. The Journal of Pain, 13(12), 11881197.

32. Spruijt, R. J., \& Wabeke, K. B. (1995). Psychological factors related to the prevalence of temporomandibular joint sounds. Journal of Oral Rehabilitation, 22(11), 803-808.
33. Ibrahim, A. K., Kelly, S. J., Adams, C. E., \& Glazebrook, C. (2013). A systematic review of studies of depression prevalence in university students. Journal of psychiatric research,47(3), 391-400.

34. Mykletun, A., Stordal, E., \& Dahl, A. A. (2001). Hospital Anxiety and Depression (HAD) scale: factor structure, item analyses and internal consistency in a large population. The British journal of psychiatry, 179(6), 540-544.

35. Al Aseri, Z. A., Suriya, M. O., Hassan, H. A., Hasan, M., Sheikh, S. A., Al Tamimi, A., ... \& Khalid, N. (2015). Reliability and validity of the Hospital Anxiety and Depression Scale in an emergency department in Saudi Arabia: a crosssectional observational study. BMC emergency medicine, 15(1), 1-6.

36. Manfredini, D., \& Lobbezoo, F. (2010). Relationship between bruxism and temporomandibular disorders: a systematic review of literature from 1998 to 2008. Oral Surgery, Oral Medicine, Oral Pathology, Oral Radiology, and Endodontology, 109(6), e26-e50.

37. Jiménez-Silva, A., Peña-Durán, C., Tobar-Reyes, J., \& Frugone-Zambra, R. (2017). Sleep and awake bruxism in adults and its relationship with temporomandibular disorders: a systematic review from 2003 to 2014. Acta Odontologica Scandinavica, 75(1), 36-58.

Cite This Article: Aymen Ben Hadj Khalifa et al (2021). Association of Temporomandibular Disorders Symptoms with Anxiety and Depression in Tunisian Patients. EAS J Dent Oral Med, 3(2), 25-30. 